\title{
Adecuación al flujograma PROA de neumonía aguda comunitaria en la emergencia del Hospital de Clínicas en 2019, Uruguay.
}

1-Stefano Fabbiani ORCID: 0000-0002-8720-7228.

2-Federico Garafoni ORCID: 0000-0002-2629-3273.

3-Mauricio Caetano ORCID: 0000-0002-5910-9612

4-Ana Karen Camacho ORCID: 0000-0002-6402-4613

5-Santiago Contrera ORCID: 0000-0003-3639-8498

6-Tatiana Fernández ORCID: 0000-0002-6402-4613

7-Valentina Fernández ORCID: 0000-0002-0808-2624

8-Javier Jara. ORCID: 0000-0002-4590-7824

9-Stephanie Viroga ORCID: 0000-0001-9065-6692

10- Noelia Speranza ORCID: 0000-0002-3330-9974.

11-José Gorrasi ORCID: 0000-0001-6308-5732

1,2-Asistente del Departamento de Farmacología y Terapéutica. Facultad de Medicina. UdelaR.

3,4,5,6,7,8-Bachiller Medicina Facultad de Medicina. UdelaR.

9-Profesor Adjunta del Departamento de Farmacología y Terapéutica.

Facultad de Medicina. UdelaR.

10-Profesor Agregado de

Departamento de Farmacología y Terapéutica. Facultad de Medicina.

UdelaR

11-Profesor Agregado del

Departamento de Emergencia. Hospital de Clínicas. Facultad de Medicina. UdelaR.

\section{Adaptation to the PROA flowchart for community acute pneumonia in the Hospital de Clínicas emergency in 2019, Uruguay. \\ Adaptação ao fluxograma PROA para pneumonia aguda comunitária na emergência do Hospital de Clínicas em 2019, Uruguai.}

Resumen: Introducción: La resistencia antimicrobiana es uno de los principales problemas de salud pública mundial. Representa una causa importante de morbilidad en la población general y un elevado costo para los sistemas sanitarios. La Neumonía Aguda Comunitaria (NAC) representa una de las principales infecciones bacterianas en nuestro medio. Objetivo general: Evaluar la adecuación al flujograma del Programa de Optimización de Antimicrobianos (PROA) para el manejo de NAC en Departamento de Emergencia del Hospital de Clínicas (HC) entre julio y agosto de 2019. Materiales y métodos: Se realizó un estudio observacional, transversal, en el período de julio-agosto de 2019, en Departamento de Emergencia del Hospital de Clínicas. Se incluyeron pacientes mayores de 18 años, que firmaron el consentimiento informado, diagnosticados con NAC, cumpliendo criterios clínicos e imagenológicos establecidos en el flujograma del PROA del Hospital de Clínicas. Se elaboró una base de datos diseñada a partir del flujograma. Resultados: Se incluyeron 51 pacientes para el análisis. La edad promedio fue 54 años, 28 eran mujeres. Las comorbilidades más prevalentes fueron: tabaquismo, consumo de pasta base de cocaína y alcoholismo, presentes en $51 \%$ de la muestra. Treinta y cinco pacientes presentaron criterios de severidad, predominando insuficiencia respiratoria en $71 \%$. Un $43 \%$ presentaron factores de riesgo para microorganismos multirresistentes. Se observó una adecuación al PROA de $41 \%$. Discusión: La adecuación al tratamiento recomendado fue inferior a la descrita en otros trabajos. El principal problema fue una errónea clasificación en los grupos de riesgo propuestos en el flujograma, ocasionando la hospitalización de pacientes que debieron recibir tratamiento ambulatorio, recibiendo antibioticoterapia de mayor espectro. Conclusiones: La existencia de PROA hospitalarios permite realizar monitoreo de prácticas diagnósticas y prescripción de antimicrobianos. Se observó una inadecuada aplicación del flujograma, lo que determinó el uso de antibióticos de mayor espectro con riesgo potencial del desarrollo de resistencia.

Palabras clave: Programa de Optimización Antimicrobiana (PROA). Neumonía Aguda Comunitaria (NAC). Resistencia antimicrobiana. Emergencia.

Abstract: Introduction: Antimicrobial resistance is one of the main world public health problems. It represents an important cause of morbidity in general population and a high cost for health systems. Community Acquired Pneumonia (CAP) represents one of the main bacterial infections in our midst. Objective: To evaluate the adequacy of the Antimicrobial Stewardship (AMS) in the management of CAP in the Emergency Department of Hospital de Clínicas $(\mathrm{HC})$ between July and August 2019. Methods: An observational, cross-sectional study was conducted from JulyAugust 2019, in the Emergency Department of Hospital de Clínicas. Patients older than 18 years old were included, who signed the informed consent, diagnosed with CAP, fulfilling clinical and imaging criteria established in the flowchart. A database designed from the AMS flow chart of the Hospital de Clínicas was developed. Results: 51 patients were included for the analysis. The average age was 54 years, 28 were women. The most prevalent comorbidities were smoking, 
consumption of cocaine paste or alcoholism, present in $51 \%$ of the sample. Thirty-five patients presented severity criteria, prevailing respiratory failure in $71 \%$. Risk factors for multiresistant microorganisms was $43 \%$. PROA adequacy of $41.2 \%$ was observed. Discussion: The adequacy to the recommended treatment was lower than that described in other papers. The main problem was an erroneous classification in the risk groups proposed in the flowchart, causing hospitalization of patients who had to receive treatment at home, receiving broader spectrum antibiotic therapy. Conclusions: The existence of hospital stewardships allows monitoring of diagnostic practices and antimicrobial prescription. Inadequate application of the flow chart was observed, which determined the use of broader spectrum antibiotics with potential risk of developing resistance.

Keywords: Antimicrobial Optimization Program (PROA). Antimicrobial stewardship. Community Acute Pneumonia (NAC). Antimicrobial resistance. Emergency.

Resumo: Introdução: A resistência antimicrobiana é um dos principais problemas de saúde pública global. Representa uma das principais causas de morbidade na população em geral e um alto custo para os sistemas de saúde. A Pneumonia Aguda Comunitária (PAC) representa uma das principais infecções bacterianas em nosso meio. Objetivo: Avaliar a adequação do fluxograma do Programa de Otimização de Antimicrobianos (PROA) para o gerenciamento do PAC no Pronto Atendimento do Hospital de Clínicas (HC) entre julho e agosto de 2019. Materiais e métodos: Foi realizado um estudo observacional, transversal, no período de julho a agosto de 2019, no Pronto-Socorro do Hospital de Clínicas. Foram incluídos pacientes maiores de 18 anos, que assinaram o termo de consentimento livre e esclarecido, com diagnóstico de PAC, que preenchessem os critérios clínicos e de imagem estabelecidos no fluxograma do PROA do Hospital de Clínicas. Um banco de dados projetado a partir do fluxograma foi desenvolvido. Resultados: 51 pacientes foram incluídos para análise. A idade média era de 54 anos, 28 eram mulheres. As comorbidades mais prevalentes foram: tabagismo, consumo de pasta base de cocaína e etilismo, presentes em $51 \%$ da amostra. Trinta e cinco pacientes apresentaram critérios de gravidade, predominando insuficiência respiratória em $71 \%$. 43\% apresentaram fatores de risco para microrganismos multirresistentes. Observou-se adequação ao PROA de $41 \%$. Discussão: A adequação ao tratamento recomendado foi inferior ao descrito em outros estudos. O principal problema era uma classificação errônea nos grupos de risco propostos no fluxograma, ocasionando a internação de pacientes que precisavam receber tratamento ambulatorial, recebendo antibioticoterapia de maior espectro. Conclusões: A existência de PROAs hospitalares permite o monitoramento das práticas diagnósticas e prescrição de antimicrobianos. Observou-se uma aplicação inadequada do fluxograma, que determinou o uso de antibióticos de maior espectro e com potencial risco de desenvolvimento de resistência.

Palavras-chave: Programa de Otimização Antimicrobiana (PROA). Pneumonia aguda comunitária (NAC). Resistência antimicrobiana. Emergência. 


\section{Introducción}

La resistencia antimicrobiana es uno de los principales problemas de salud pública a nivel mundial. El uso inapropiado de antibióticos, contribuye a su desarrollo y perpetuación. A esto se suma la poca inversión y desarrollo de nuevos antimicrobianos por parte de la industria farmacéutica. La resistencia antimicrobiana genera mayor morbilidad, mortalidad y aumento de los costos de atención en salud. ${ }^{1}$

Como respuesta a esta situación, se han desarrollado distintas estrategias para contener la progresión de dicha problemática entre las que se encuentran los "Programas de Optimización de Antimicrobianos (PROA)". ${ }^{1-7}$

En 1997 en la Unión Europea, Austria implementó uno de los primeros programas para racionalizar el uso de antibióticos. Un año más tarde, la Organización Mundial de la Salud (OMS) emitió una recomendación para que todos los países desarrollarán intervenciones y políticas nacionales al respecto. ${ }^{8}$

En Uruguay se han desarrollado desde hace varios años diferentes esfuerzos en este sentido. En el Hospital de Clínicas "Dr. Manuel Quintela" (HC) en el año 2018 se creó un PROA con el objetivo de optimizar el uso de los antibióticos, prevenir el desarrollo de nuevas resistencias y contener las ya conocidas, mejorar la evolución clínica de los pacientes, disminuir la morbimortalidad y disminuir los costos derivados del uso inadecuado de los antimicrobianos. ${ }^{9,10}$

La primera medida en instaurarse fue la creación de flujogramas de diagnóstico y tratamiento de patologías infecciosas más prevalentes a nivel hospitalario: neumonía aguda comunitaria (NAC), infecciones urinarias, infecciones de piel y partes blandas e infecciones intraabdominales. Estas fueron elaboradas por un equipo multidisciplinario. ${ }^{11,12}$

Se ha observado en estudios internacionales que la intervención de un equipo multidisciplinario de utilización de antimicrobianos en comparación con el empleo de guías generales de antibióticos empíricos, mejora significativamente la prescripción apropiada de estos en un 94$95 \%$. Esta capacitación debe ser longitudinal, repetitiva y basada en el patrón poblacional. ${ }^{12,13}$

Una de las medidas más importantes para medir el impacto evolutivo de este tipo de Programas es conocer el grado de cumplimiento a sus recomendaciones.

El objetivo de este estudio es evaluar la adecuación y cumplimiento del flujograma del PROA del Hospital de Clínicas para el manejo de NAC en el Departamento de Emergencia del Hospital, en el período julio-agosto de 2019.

\section{Material y métodos}

Se realizó un estudio observacional, transversal, entre junio y agosto del 2019, en el Departamento de Emergencia del Hospital de Clínicas.

Se incluyeron pacientes mayores de 18 años, que accedieron a participar en el estudio mediante la firma del consentimiento informado y que fueron diagnosticados con NAC. Se definió NAC si el paciente presentaba sintomatología menor a 5 días de duración previo a la consulta de: fiebre (temperatura $\geq$ a $37,7^{\circ} \mathrm{C}$ axilar) y alguno de los siguientes síntomas: tos, expectoración, disnea, dolor torácico o crepitantes. A su vez, debían contar con un estudio de imagen torácico que mostrara un infiltrado nuevo sin diagnóstico alternativo (referencia de la definición de neumonía).

Se excluyeron los pacientes institucionalizados y aquellos pacientes que presentaron una internación en las 72 horas previas a la consulta actual y embarazadas.

\section{a) Metodología}

Diariamente, parte del grupo de investigadores concurrieron al Departamento de Emergencia en búsqueda de pacientes que cumplieran con los criterios de inclusión y quisieran participar del estudio. Previa firma del consentimiento informado se procedió a analizar la historia de manera manual y obtener los datos para completar la planilla de recolección de datos prediseñada.

Se recabaron de la historia clínica datos patronímicos: edad y sexo. Las comorbilidades evaluadas fueron: alergia, diabetes mellitus, asplenia, insuficiencia cardiaca, enfermedad hepática, enfermedad renal, alcoholismo, tabaquismo, consumo de pasta base, enfermedad 
pulmonar obstructiva crónica (EPOC), asma, enfermedad neurológica, colagenopatía, inmunodeficiencia patológica o por fármacos, neoplasias.

Se evaluaron los siguiente criterios de severidad: insuficiencia respiratoria, saturación de oxihemoglobina menor a 93\%, neumonía necrotizante, intolerancia digestiva, dos o más lóbulos pulmonares afectados, derrame pleural, inestabilidad hemodinámica, fracaso antibiótico previo, score qSOFA mayor igual a 2.

Para definir a que grupo de riesgo del PROA se encontraba cada paciente, se realizó una tabla de recolección de datos que incluía edad, sexo, comorbilidades, criterios de severidad, factores de riesgo para microorganismos resistentes, score CURB-65. A partir de la misma se realizó una clasificación por parte de los investigadores en los grupos de riesgo según el flujograma del PROA.

Se consideraron factores de riesgo para infección por Pseudomonas aeruginosa: internación mayor a 7 días, bronquiectasia, fibrosis quística, EPOC con volumen de espiración forzada en un segundo menor a 30, consumo de prednisona mayor a $10 \mathrm{mg}$ en las últimas dos semanas, administración reciente de antibióticos en los últimos tres meses o cuatro o más en el año e inmunodepresión severa; para enterobacterias con betalactamasas de espectro extendido (BLEE): antibioticoterapia previa en los últimos tres meses (quinolonas, carbapenemes y betalactamasas), diabetes mellitus, inmunodepresión; y para Staphylococcus aureus meticilino resistente (SAMR): jóvenes sanos con NAC severa y rápidamente progresiva y/o hemoptisis, infecciones de piel y partes blandas, usuarios de drogas intravenosas, neumonía necrotizante, infecciones o colonizaciones previas por SAMR o Staphylococcus aureus meticilino sensible (SAMS), personas privadas de libertad.

Se realizó el diagnóstico mediante la realización de estudios de imagen, incluyéndose tanto la radiografía de tórax como la tomografía computada. También se tuvieron en cuenta los hemocultivos, cultivo de la expectoración y/o antígeno neumocóccico en orina en busca del patógeno causal.

La variable primaria fue adecuación al flujograma del PROA para NAC, entendiendo como tal si se cumplía para las siguientes tres características: clasificación de riesgo del paciente, tratamiento antimicrobiano según grupo de riesgo correspondiente y posología. De no cumplir al menos una de las tres se consideró adecuación parcial y si no había adecuación a ninguna de las 3 se consideró no adecuación.

Los flujogramas se encontraban disponibles en el Departamento de Emergencia, al acceso de todo el equipo asistencial.

\section{b) Análisis estadístico}

Las variables se expresaron como valores absolutos y porcentajes.

El análisis se realizó mediante Epiinfo versión 3.5.3, utilizando estadística descriptiva.

c) Ética

El proyecto fue aprobado por el Comité de Ética del Hospital de Clínicas.

\section{Resultados}

De los 53 pacientes que cumplían con los criterios de inclusión, 51 integraron el análisis dado que dos no aceptaron participar en el estudio. En la tabla 1 se presentan las características epidemiológicas de la población analizada.

\begin{tabular}{|l|l|}
\hline \multicolumn{1}{|c|}{ Edad promedio } & \multicolumn{1}{c|}{$\mathbf{5 4}(\mathbf{1 8 \% )}$} \\
\hline Sexo & \\
\hline Femenino & $28(55 \%)$ \\
\hline Masculino & $23(45 \%)$ \\
\hline Comorbilidades & \\
\hline $\mathrm{Si}$ & $43(84 \%)$ \\
\hline $\mathrm{No}$ & $8(16 \%)$ \\
\hline
\end{tabular}


Tabla 1: Características de la población $\mathrm{n}=51$

Tabla 2: Factores de riesgo para microorganismos específicos

Tabla 3: Criterios de severidad de la

\begin{tabular}{|l|l|}
\hline Tabaquismo, PBC, Alcoholismo & $22(51 \%)$ \\
\hline EPOC o asma & $19(19 \%)$ \\
\hline Inmunodeprimidos & $9(21 \%)$ \\
\hline Otros & $18(42 \%)$ \\
\hline PBC: pasta base de cocaína. EPOC: enfermedad pulmonar obstructiva crónica. \\
\hline
\end{tabular}

Un 45\% de la población presentaba factores de riesgo para microorganismos multirresistentes. (Tabla 2)

\begin{tabular}{|l|l|}
\hline $\mathrm{Si}$ & $23(45 \%)$ \\
\hline No & $28(55 \%)$ \\
\hline P. aeruginosa & $11(22 \%)$ \\
\hline BLEE & $18(35 \%)$ \\
\hline SAMR & $4(8 \%)$ \\
\hline
\end{tabular}

Todos los pacientes contaban con estudio imagenológico de confirmación del diagnóstico clínico presuntivo de NAC (en su totalidad radiografías de tórax). Un $69 \%$ de los pacientes presentaban criterios de severidad, siendo la insuficiencia respiratoria el más frecuente $(71 \%)$. Tabla 3.

\begin{tabular}{|l|l|}
\hline $\mathrm{Si}$ & $35(69 \%)$ \\
\hline No & $16(31 \%)$ \\
\hline Insuficiencia respiratoria & $25(71 \%)$ \\
\hline 2 o más lóbulos pulmonares afectados & $11(31 \%)$ \\
\hline Derrame pleural & $10(29 \%)$ \\
\hline Otros & $15(42 \%)$ \\
\hline
\end{tabular}

Se solicitaron hemocultivos a 25 pacientes, de los cuales 4 fueron positivos. Nueve pacientes contaron con un cultivo de expectoración, de los cuales 2 fueron positivos, con el hallazgo de diplococos Gram positivos. Se buscó antígeno neumocócico en orina a 9 pacientes, siendo 1 positivo.

Se clasificaron 9 pacientes en el grupo 1 (18\%, 1a: 3 y 1b: 6), 37 pacientes en el grupo 2 (72\%, 2a: 20 y 2 b: 17) y 5 pacientes en el grupo 3 (10\%, 3a: 1 y $3 b: 4)$.

A partir de estos grupos de riesgo y la presencia de factores de riesgo para mala evolución se evaluó si el tratamiento antibiótico instaurado se adecuaba o no al flujograma.

De los 51 pacientes, se encontró tratamiento adecuado en 21 pacientes (41\%), no adecuado en 2 pacientes (4\%), y parcialmente adecuado en 28 pacientes (55\%)

En la tabla 4 se presenta el número de errores considerando la clasificación, la elección del antibiótico y la posología.

\begin{tabular}{|c|c|c|c|c|c|}
\hline \multicolumn{2}{|c|}{1 error } & \multicolumn{2}{|l|}{2 errores } & \multicolumn{2}{|c|}{3 errores } \\
\hline Clasificación & 5 & Clasificación + antibiótico & 21 & $\begin{array}{l}\text { Clasificación } \\
+ \\
\text { antibiótico } \\
+ \\
\text { posología }\end{array}$ & 2 \\
\hline Antibiótico & - & Antibiótico + posología & - & - & - \\
\hline Posología & 2 & Clasificación + posología & - & - & - \\
\hline
\end{tabular}

El antibiótico más utilizado en el tratamiento la NAC fue ampicilina-sulbactam en 43 pacientes (sólo o en combinación con otros antibióticos), representando el $84,3 \%$ del total de los antibióticos utilizados. 
Del total de pacientes se encontró que $34(66.7 \%)$ pacientes recibieron tratamiento en monoterapia: 30 recibieron tratamiento con ampicilina sulbactam, 2 con piperacilina tazobactam y 2 con levofloxacina.

17 pacientes iniciaron con antibioticoterapia combinada 15 con dos antibióticos y 2 con tres antibióticos. La combinación más frecuente de dos antibióticos fueron ampicilina-sulbactam + claritromicina en 8 pacientes (Tabla 5).

\begin{tabular}{|l|l|l|l|l|l|}
\hline \multicolumn{2}{c}{$\begin{array}{c}\text { Monoterapia } \\
\text { N= 34 }\end{array}$} & \multicolumn{1}{c|}{$\begin{array}{c}\text { Biterapia } \\
\text { N= 15 } \\
\text { Ne }\end{array}$} \\
\hline SAM & 30 & SAM + Claritromicina & 8 & $\begin{array}{l}\text { Ceftriaxona + SAM + } \\
\text { Claritromicina }\end{array}$ & 1 \\
\hline PTZ & 2 & PTZ + Claritromicina & 2 & SAM + PTZ + Ceftriaxona & 1 \\
\hline Levofloxacina & 2 & SAM + TMP/SMX & 2 & & \\
\hline & & Otros varios & 3 & & \\
\hline
\end{tabular}

Si bien no fue una variable de estudio del trabajo, se evidenció una rotación del plan antibiótico empírico inicial intra-tratamiento en 4 pacientes durante su estadía en la emergencia, de los cuales, 1 fue modificado debido a la carencia del antibiótico requerido en la farmacia del Hospital, para el resto no constaba en la historia el motivo de rotación del antimicrobiano.

\section{Discusión}

Este trabajo es el primero en evaluar el impacto en la práctica clínica de los flujogramas del PROA del Hospital de Clínicas. Se observó una buena predisposición por parte de los pacientes al participar en la investigación, dado que 51 de los 53 candidatos, accedieron a participar de la misma.

Según la literatura, la NAC se presenta con mayor frecuencia en adultos mayores de 65 años, siendo este un factor de riesgo independiente, con un patrón estacional concentrándose en los meses de invierno. La edad promedio se encuentra en torno a los 56 años, presentando la población de nuestro estudio un promedio de 54 años, próximo a la incidencia reportada. ${ }^{14-16}$ La incidencia fue mayor en mujeres, siguiendo la incidencia nacional de neumonía según datos de $2019 .{ }^{17}$

La comorbilidad más prevalente fue el consumo de alcohol, pasta base de cocaína o tabaco, que se encuentran agrupadas en la misma categoría, lo que dificulta conocer cuál de ellas es la más prevalente en esta muestra, seguido de EPOC. Todos ellos son factores de riesgo y comorbilidades para una mala evolución, estando presentes en la mitad de la muestra, siendo muy frecuente su asociación con el desarrollo y severidad de NAC.

Todos los pacientes contaron con estudio imagenológico, siendo este un pilar fundamental en el diagnóstico de NAC. Los cultivos microbiológicos no fueron solicitados según la indicación del flujograma, por lo tanto, si bien este dato no fue una variable a estudiar se considera también una no adecuación al mismo.

De acuerdo al flujograma, a los pacientes clasificados a partir del grupo 2 (42 pacientes) le correspondía la realización de un hemocultivo, pero según nuestros datos, eran sólo 36 los pacientes que presentaban criterios para su solicitud.

La adecuación encontrada en este trabajo fue inferior a la descrita en estudios citados previamente y se relaciona principalmente a la clasificación errónea según lo indicado en el flujograma o por la selección del antibiótico. ${ }^{6,7}$ En la mayoría de estos pacientes la selección del antibiótico fue de mayor espectro, siendo coincidente con el flujograma.

Un motivo posible del por qué los médicos no clasificaron a los pacientes en las categorías, podría estar vinculado a la mayor confianza en su criterio clínico que en el seguir las recomendaciones del flujograma.

En cuanto a la selección del antibiótico, la ausencia de programas de entrenamiento protocolizado en uso de antibióticos con evaluación de sus resultados, puede ser una causa a considerar. Este estudio aporta por sus resultados la necesidad de implementar estas actividades. 
Una clasificación inadecuada, con una incorrecta administración de antibióticos (mayor espectro al indicado), es un importante factor, ya que determina la necesidad de internación del paciente para tratamiento intravenoso sin un mayor beneficio para el usuario, determinando posiblemente mayores gastos de recursos y un potencial aumento de la resistencia antimicrobiana.

Otra posible causa de no adecuación, como se describe en la literatura, puede deberse a la dinámica de organización de la emergencia y la alta tasa de rotación de personal con distintos criterios de selección de antibióticos, sin continuar de forma longitudinal al paciente y su evolución ${ }^{(18)}$

Un aspecto a destacar es que el flujograma no contempla el patrón evolutivo del paciente dado que se trata de una herramienta estática y solo orienta al médico al inicio del tratamiento, dando como resultado que una variación en la situación clínica del paciente provoque un cambio en la terapéutica que puede no estar sujeto a las pautas del PROA.

Según el flujograma el uso de ampicilina-sulbactam se recomienda desde el grupo 2a, pudiendo considerarse amoxicilina-clavulánico/amoxicilina-sulbactam como equivalentes, desde el grupo 1b. Contando los pacientes en ambos grupos, se debió haber utilizado ampicilina-sulbactam en 26 pacientes (51\%), sin embargo, el uso fue más amplio alcanzando el $84 \%$. Se observó que en la mayoría de los tratamientos instaurados no se contemplaron las características propias del paciente, como comorbilidades y criterios de severidad que presentaron, generándose así, el uso de ampicilina sulbactam como una práctica frecuente, práctica que no se adecua a las recomendaciones del PROA. Sería necesario contar con mayor información para entender cómo se justificó la salida del protocolo.

Múltiples estudios han demostrado que la aplicación de protocolos y guías, en conjunto con educación médica continua y la integración del equipo de salud con un grupo referente en optimización de antibióticos, determinan una mejoría significativa en la aplicación de la antibioticoterapia y una reducción de los días de tratamiento y estancia hospitalaria, determinando a su vez una reducción de los costos asistenciales. ${ }^{5,7,19-22}$ Los PROA contribuyen a lograr estos objetivos asistenciales y de uso racional de medicamentos.

La principal limitante de este estudio fue que los resultados se obtuvieron mediante la revisión manual de las historias clínicas, en las cuales se encontró un registro heterogéneo de los criterios clínicos para establecer la clasificación de riesgo y el porqué de la selección de los antibióticos prescritos.

La falta de historia clínica electrónica de manera sistemática es un factor que quizás impidió un mejor cumplimiento y un mejor registro de las actividades llevadas a cabo por los médicos de la emergencia. La misma permitiría, por un lado, un registro longitudinal y dinámico y facilitar la clasificación de los pacientes, hacerlas más automatizadas y aún obligatorias para los grupos de riesgo, evitando así depender de la voluntad y criterio subjetivo de los médicos. Esto facilitaría la comunicación interdisciplinaria y generaría una retroalimentación con el equipo tratante del paciente. Sería interesante evaluar el impacto de la adecuación o no al flujograma sobre el resultado clínico final de los pacientes.

\section{Conclusiones}

La existencia de PROA hospitalarios permite realizar evaluaciones y monitoreo de las prácticas diagnósticas y de prescripción de antimicrobianos. Se observó una inadecuada aplicación del flujograma de NAC que determinó el uso de antibióticos de mayor espectro con el riesgo potencial del desarrollo de resistencia antimicrobiana.

Este estudio puede servir de insumo para planificar estrategias de mejora para lograr mayor adecuación del flujograma en el Departamento de Emergencia del Hospital de Clínicas.

\section{Agradecimientos}

A los médicos del Departamento de Emergencia del Hospital de Clínicas, en particular a Prof. Dr. Fernando Machado por haber autorizado a realizar la recolección de datos en el servicio.

A los integrantes del PROA del Hospital de Clínicas, Prof. Julio Medina, Prof Adj. Henry Albornoz, Prof. Adj. Martín López, Q.F. Gabriela Díaz, Prof. Adj. Dra. Pilar Gadea, Asist. Karina Nuñez, Prof. Agda. Fernanda Blasina y Dr. Juan Siri y especialmente a la Dra. Daniela Paciel por sus invalorables aportes. 


\section{Bibliografía}

1- Centro CDS. Estrategia mundial de la OMS para contener la resistencia a los antimicrobianos. Rev Panam Salud Publica/Pan Am J Public Heal. 2001;10(4):284-94.

2- Denny KJ, Gartside JG, Alcorn K, Cross JW, Maloney S, Keijzers G. Appropriateness of antibiotic prescribing in the Emergency Department. J Antimicrob Chemother. 2019;74(2):515-20.

3- Allerberger F, Lechner A, Wechsler-Fördös A, Gareis R. Optimization of antibiotic use in hospitals Antimicrobial stewardship and the EU Project ABS International. Chemotherapy. 2008;54(4):260-7. 14

4- Pulcini C, Binda F, Lamkang AS, Trett A, Charani E, Goff DA, et al. Developing core elements and checklist items for global hospital antimicrobial stewardship programmes : a consensus approach. Clin Microbiol Infect. 2019;22(11):3702-11.

5- Medina-Morales DA, Machado-Duque ME, Machado-Alba J. Resistencia a antibióticos, una crisis global. Rev Médica Risaralda. 2015;21(1):74-74.

6- Burgess LH, Miller K, Cooper M, Moody J, Englebright J, Septimus E. Phased implementation of an antimicrobial stewardship program for a large community hospital system. Am J Infect Control. 2019;47(1):69-73.

7- Cisneros JM, Neth O, Gil-Navarro MV, Lepe JA, Jiménez-Parrilla F, Cordero E, et al. Global impact of an educational antimicrobial stewardship programme on prescribing practice in a tertiary hospital centre. Clin Microbiol Infect. 2014;20(1):82-8.

8- Camins BC, King MD, Wells JB, Googe HL, Patel M, Kourbatova EV, et al. The Impact of an Antimicrobial Utilization Program on Antimicrobial Use at a Large Teaching Hospital: A Randomized Controlled Trial. Infect Control Hosp Epidemiol. 2009;30(10):931-938.

9- Nasr Z, Babiker A, Elbasheer M, Osman A, Elazzazy S, Wilby KJ. Practice implications of an antimicrobial stewardship intervention in a tertiary care teaching hospital, Qatar. East Mediterr Heal J. 2019;25(3):172-80.

10- Universidad de la República, Facultad de Medicina, Hospital de Clínicas. Programa de Optimización de Antimicrobianos del Hospital de Clínicas: documento Marco de funcionamiento del PROA [Internet] Montevideo: PROA, 2018 [acceso: 31/03/2021]. Disponible en: https://www.proa.hc.edu.uy/images/ documento_marco.pdf

11- Leung E, Weil D, Raviglione M, Nakatani $\mathrm{H}$. The WHO policy package to combat antimicrobial resistance. Bull WHO. 2011; 89:390-392. doi: 10.2471/BLT.11.08843

12- Mateos S, Añón X, López M, Susana Cabrera A, Sosa L, Pereira G, et al. Rational use of antibiotics in the department of internal medicine from a university hospital: Results of a pilot experience. Rev Chil Infectol. 2012;29(1):7-13.

13- Universidad de la República, Facultad de Medicina, Hospital de Clínicas. Guías y flujogramas del Programa de Optimización de Antimicrobianos del Hospital de Clínicas [Internet]. Montevideo: PROA, 2018 [acceso: 31/03/2021] Disponible en: https://www.proa.hc.edu.uy/index.php?option=com_content \&view=article\&id=23:infecciones-de-piel-y-partes-blandas\&catid=15:guias-y-flujogramas

14- Valdivia G. Epidemiología de la neumonía del adulto adquirida en la comunidad. Rev Chil Infect 2005; 22 (Supl 1): S11-S17.

15- Vacarezza C. Vázquez R. Bartesaghi L. Lerena V. Perdomo J. Tondo A et al. Neumonía aguda del adulto adquirida en la comunidad. Ensayo terapéutico controlado: Uruguay. Arch. Med Int. 2010, vol.32, n.2-3. Disponible en: http://www.scielo.edu.uy/scielo.php?script=sci_arttext\&pid=S1688423X2010000200003\&lng=es.

16- Irizar MI, Arrondo MA, Insausti MJ, Mujica J, Etxabarri P, Ganzarain R. Epidemiología de la neumonía adquirida en la comunidad. Aten Primaria.2013;45(10):503-513. DOI: 10.1016/j.aprim.2013.05.003

17- Uruguay. Ministerio de Salud Pública, Departamente de Vigilancia en Salud. Morbilidad por Enfermedades No Transmisibles. Uruguay, diciembre 2019. Montevideo: MSP, 2019.

18- Díaz A, Calvo M, O’Brien A, Farías G, Mardónez JM, Saldías F. Utilidad clínica de los hemocultivos en pacientes hospitalizados por neumonía adquirida en la comunidad. Rev. méd. Chile. 2002 vol.130, n.9 http://dx.doi.org/10.4067/S0034-98872002000900005.

19- Seija V, Vignoli R. Control de poblaciones microbianas. En: Universidad de la República, Facultad de Medicina, Instituto de Higiene, Departamento de Bacteriología y Virología, coord. Temas de Bacteriología y Virología médica. $3^{\mathrm{a}}$ ed. Montevideo: 3era edición. Oficina del Libro FEFMUR; 2006. 631-648 p. 
20- CDC. Antibiotic Use in the United States, 2017: Progress and Opportunities. Atlanta, GA: US Department of Health and Human Services, CDC; 2017.

21- Galanter KM, Ho J. Impact of an Empiric Therapy Guide on Antibiotic Prescribing in the Emergency Department. J Hosp Infect. 2019.

22- Guan X, Tian Y, Song J, Zhu D, Shi L. Effect of physicians' knowledge on antibiotics rational use in China's county hospitals. Soc Sci Med. 2019; 224: 149-55.

\section{Aportes cada autor al trabajo}

Stefano Fabbiani: Concepción y diseño del trabajo. Análisis e interpretación de los datos o resultados. Redacción del manuscrito. Revisión crítica del manuscrito.

Federico Garafoni: Concepción y diseño del trabajo. Análisis e interpretación de los datos o resultados. Redacción del manuscrito. Revisión crítica del manuscrito.

Mauricio Caetano: Recolección de datos o realización de los experimentos. Análisis e interpretación de los datos o resultados.

Ana Karen Camacho: Recolección de datos o realización de los experimentos. Análisis e interpretación de los datos o resultados.

Santiago Contrera: Recolección de datos o realización de los experimentos. Análisis e interpretación de los datos o resultados.

Tatiana Fernández: Recolección de datos o realización de los experimentos. Análisis e interpretación de los datos o resultados.

Valentina Fernández: Recolección de datos o realización de los experimentos. Análisis e interpretación de los datos o resultados.

Javier Jara: Recolección de datos o realización de los experimentos. Análisis e interpretación de los datos o resultados.

Stephanie Viroga: Concepción y diseño del trabajo. Análisis e interpretación de los datos o resultados. Redacción del manuscrito. Revisión crítica del manuscrito.

Noelia Speranza: Concepción y diseño del trabajo. Análisis e interpretación de los datos o resultados. Redacción del manuscrito. Revisión crítica del manuscrito.

José Gorrasi: Análisis e interpretación de los datos o resultados. Redacción del manuscrito. Revisión crítica del manuscrito. 